\title{
Study of new onset cutaneous manifestations in Rheumatic Diseases
}

\author{
Sonawale A. ${ }^{1}$, Sabnis N. ${ }^{2}$, Bankar N. ${ }^{3}$ \\ ${ }^{1}$ Dr. Archana Sonawale, Associate Professor, ${ }^{2}$ Dr. Nilakshi Sabnis, Assistant Professor, ${ }^{3}$ Dr. Nirmal Bankar, Resident; \\ all authors are affiliated with Department of Medicine, Seth G.S.M.C. \& K.E.M.H, Mumbai, Maharashtra, India.
}

Corresponding Author: Dr. Nilakshi Sabnis, Assistant Professor, Department of Medicine, Seth GS Medical College \& KEMH, Parel, Mumbai, India. E-mail: sabnis.nilakshi@gmail.com

\begin{abstract}
Background: Skin manifestations are an important clue to underlying rheumatological conditions and at times the first manifestation of the disease. Their identification helps in diagnosis, classification and follow up of these diseases. Hence we conducted this study to assess the new onset cutaneous lesions in patients with rheumatic diseases and correlate skin lesions with disease activity and study the response to therapy over a period of 3 months. Materials and Methods: This prospective observational study was done in KEM Hospital, Mumbai over 18 months recruiting 78 patients, presenting to Rheumatology OPD / wards with new onset skin manifestations. Disease activity was calculated as per standard indices for each rheumatological disease. Skin lesions appearing due to adverse effects of drugs or unrelated to the disease were excluded from the study. The outcome of the skin lesions was assessed at 3 months follow up. Results: Mean age of patients was 38 years with $91 \%$ being females. SLE was the most common diagnosis. The most common skin lesions were malar rash among SLE patients; rheumatoid nodules in patients of RA; Sclerodactyly in the Scleroderma patients and Heliotrope rash amongst the dermatomyositis patients. The mean SLEDAI score in the group with LE non -specific lesions was significantly higher compared to the group with LE-specific lesions $(\mathrm{P}<0.0001)$. At 3 months there was statistically significant reduction in SLEDAI score after treatment in SLE patients. In patients of RA, 74\% patients showed reduction in DAS 28 ESR score with treatment at 3 months. Systemic sclerosis patients failed to show significant improvement in Modified Rodnan's Skin Score after 3 months of treatment. Conclusions: Among all rheumatological conditions SLE presents most often with skin involvement. Patients with LE specific lesions have lower disease activity (SLEDAI score) as compared to LE nonspecific lesions. At 3 months follow up the response to treatment is good in SLE patients with reduction in SLEDAI scores and also in RA patients with reduction in DAS 28 ESR scores.
\end{abstract}

Keywords: Systemic Lupus Erythematosus, Rheumatoid arthritis, SLEDAI Score, Scleroderma, Skin Manifestations

\section{Introduction}

Skin manifestations are an important feature in most rheumatological diseases and at times provide a clue to clinch the diagnosis if detected early. In certain Rheumatological diseases like SLE the skin lesions are a part of the diagnostic clinical criteria [1,2] and help in classifying the disease and also monitor for disease activity [3].

Due to its aesthetic function, chronic and scarring or disfiguring skin lesions may have a negative impact on the quality of life and psychosocial wellbeing of the patient [4]. The worsening of the skin manifestations or appearance of new lesions can alert the physician to worsening disease activity [5].

Manuscript received: $2^{\text {nd }}$ December 2018

Reviewed: $12^{\text {th }}$ December 2018

Author Corrected: $18^{\text {th }}$ December 2018

Accepted for Publication: 24 ${ }^{\text {th }}$ December 2018
The resolution of these skin lesions with treatment also provides an easy way to visually assess response to treatment and disease activity. With the advent of biologics and other newer targeted therapies in rheumatology the skin may be secondarily involved due to adverse events or even infections [6]. Many factors such as genetic, environmental, disease activity influence the incidence and progression of the skin lesions leading to a wide variety of manifestations to be studied [7,8]. Not many studies have been published from India or other south Asian countries describing the wide variety of skin lesions in the different Rheumatological diseases. Hence we conducted a study to evaluate the various skin manifestations in rheumatological diseases presenting to a tertiary care center in western India, their prevalence, response to treatment and natural progression over 3 months. 
We included patients of 4 major Rheumatological diseases namely: Systemic Lupus Erythematosus (SLE), Rheumatoid Arthritis (RA), Systemic Sclerosis and Dermatomyositis. These patients were followed up after 3 months of treatment to see the response to therapy and also monitored for disease activity .

We tried to correlate the various skin lesions in each group with the disease activity to understand the clinical implications of these skin manifestations.

Objectives: 1) To study the new onset cutaneous lesions in patients with rheumatic diseases in rheumatology clinic or medicine wards. 2) To co-relate the skin lesions with the diagnosis and classification of underlying rheumatic disease and disease activity. 3) To assess the outcome, response to therapy and natural course of the skin lesion over a period of 3 months.

\section{Materials and Methods}

Place of study: Rheumatology clinic and medicine wards of a tertiary care Hospital in Mumbai, Maharashtra, India

Type of study: Prospective, Observational and Crosssectional study conducted over a period of 18 months.

Sampling Method and Sample size: Random sampling method was used and all successive patients presenting fulfilling the inclusion criteria were included in the study. Total 78 such patients were a part of the study sample.

Inclusion Criteria: Patients with new onset skin lesions (presenting within last 4 weeks) in cases of rheumatological diseases with age $>18$ years and willing to give written informed consent were included. Rheumatic diseases included in the study were: Systemic lupus erythematosus, Dermatomyositis, Systemic sclerosis, Rheumatoid arthritis.

Exclusion criteria: 1) Primary skin lesions not related to underlying rheumatic disease and appearing as a side effect of immunosuppressive therapy. 2) Pregnant or lactating patients.
Statistical Analysis: Descriptive data will be represented as mean +/- standard deviation, median will be used for continuous variables. The data was analysed using appropriate statistical tests like Chi-square test, paired $\mathrm{t}$ test, Spearson rho calculator wherever applicable. The $\mathrm{p}$ value of $<0.05$ was considered statistically significant.

Study Procedure: After obtaining Institutional Ethics Committee approval, 78 patients fulfilling the Inclusion criteria, willing to give informed consent were included in the study. Standard Diagnostic criteria were used to classify the patients presenting for the first time with a new onset skin lesion (onset less than 4 weeks) into the 4 rheumatic diseases included in our study.

ACR Classification criteria were applied for patients of SLE [1]; 2010 ACR/ EULAR criteria were used for Rheumatoid arthritis patients [9], Systemic Sclerosis and Dermatomyositis were diagnosed clinically as per the Rheumatologists opinion. Detailed history and clinical examination was done and relevant investigations were sent. Apart from routine tests like $\mathrm{CBC}$, Liver function tests, Kidney function tests, Urine Routine examination, ESR, CRP, Fasting and Post prandial Blood sugars, other special tests like ANA, Anti-dsDNA, 24 hour urine protein, Rheumatoid Factor, Anti-CCP Antibody, complement c3 \& c4 levels, Thyroid function tests, X-rays of Joints, etc were done as needed.

MRI or Ultrasound of Joints was done if indicated. Skin Biopsy was done as per Rheumatology and Dermatology opinion if required. Disease activity in Rheumatic diseases was calculated using objective scales like SLEDAI 2K index and SLICC ACR damage index for SLE [10,11], DAS 28 ESR for rheumatoid arthritis [12] and Modified Rodnan's Skin Score of Systemic sclerosis [13].

Lesions in SLE patients were grouped as LE Specific vs LE Non-specific (Gilliam Classification) [14]. Similarly the Lesions in RA patients were grouped as RA Specific and RA Non-Specific [15]. Improvement in skin manifestations and disease activity was monitored at the 3 months follow up visit after Standard treatment.

\section{Results}

The mean age of patients was $38 \pm 10.4$ years with the majority of the patients $(33 \%)$ being from age group $31-40$ years.Out of the 78 patients 71 patients $(91 \%)$ were females and only 7 patients were male with female to male ratio of 10: 1 . 
Original Research Article

Table-1: Rheumatological diseases included in the study and their sex distribution in the study population

\begin{tabular}{|l|c|c|c|}
\hline Rheumatic disease & Females & Males & Total (n=78) \\
\hline SLE & 42 & 4 & $46(59 \%)$ \\
\hline Rheumatoid arthritis & 19 & 4 & $23(30 \%)$ \\
\hline Systemic sclerosis & 4 & 1 & $5(6 \%)$ \\
\hline Dermatomyositis & 4 & 0 & $4(5)$ \\
\hline
\end{tabular}

As shown in Table 1, SLE was the most common diagnosis (59\%) followed by RA. All the diseases showed a female preponderance.

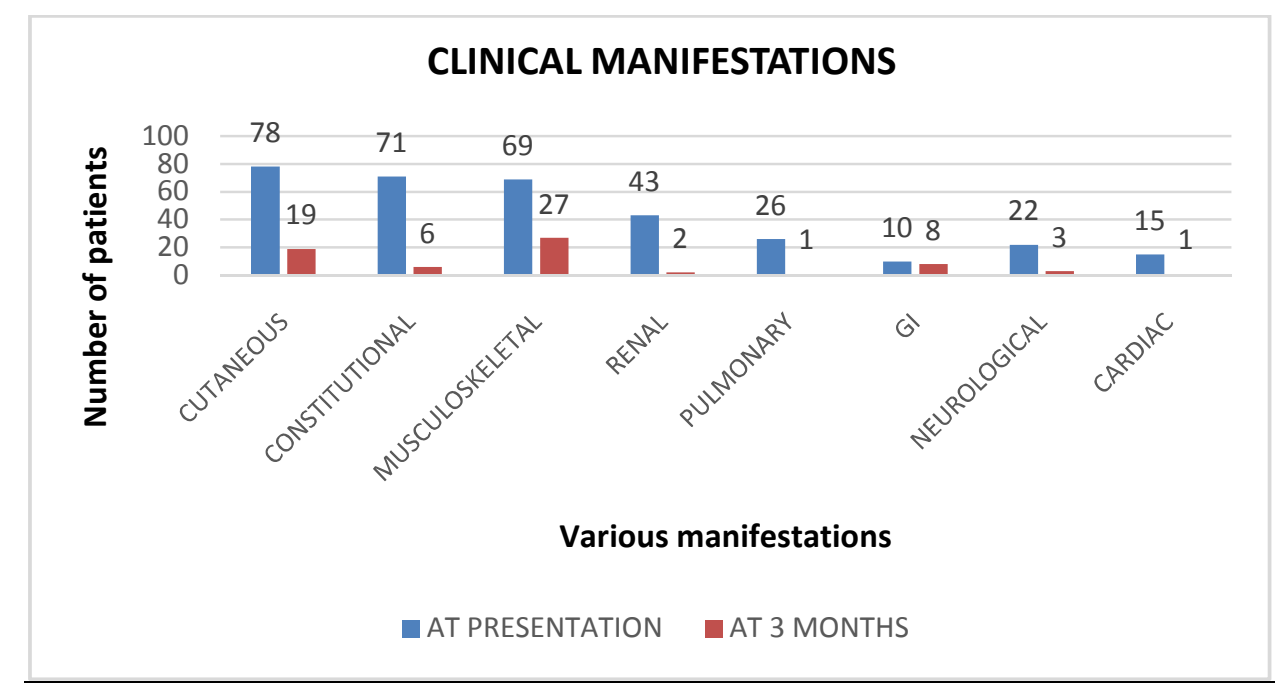

Figure-1: Clinical manifestations of the study population at presentation and at 3 months $(\mathrm{n}=78)$

Skin manifestations which were seen in all the patients included in the study at presentation ( $\mathrm{n}=78$ ), on follow up at 3 months were seen only in 24\% $(n=19)$ patients (Figure 1). Constitutional and musculoskeletal were other common symptoms at presentation, found in $91.02 \%$ and $88 \%$ patients respectively. At 3 months, musculoskeletal were the most common symptoms observed. In our study, the most common skin lesions were malar rash, found in $80 \%$ of SLE patients; rheumatoid nodules found in $86 \%$ of RA patients; Sclerodactyly, found in $80 \%$ of the SSc patients and Heliotrope rash, found in $75 \%$ of the dermatomyositis patients.

Systemic Lupus Erythematosus: SLE patients were divided by their skin manifestations into three groups having, at the time of the examination: (1) only LE-specific lesions, seen in 59\% patients ( $n=27)$; (2) only LE-nonspecific lesions, seen in $6 \%$ patients $(n=3)$ and $(3)$ both types of lesion simultaneously, seen in $35 \%$ patients $(n=16)$.

Table-2: Distribution of skin lesions seen in SLE patients as SLE specific vs SLE Nonspecific

\begin{tabular}{|l|c|c|}
\hline SLE specific skin lesions & Type of Lesion & Number of patients \\
\cline { 2 - 3 } & Malar rash & $43(93 \%)$ \\
\cline { 2 - 3 } & Photosensitivity & $21(46 \%)$ \\
\cline { 2 - 3 } & Oral ulcers & $39(84 \%)$ \\
\cline { 2 - 3 } & Discoid rash & $18(39 \%)$ \\
\cline { 2 - 3 } & Alopecia & $39(84 \%)$ \\
\hline \multirow{4}{*}{ SLE NonspecificLesions } & Lupus profundus & $3(6 \%)$ \\
\cline { 2 - 3 } & Type of Lesion & Number of patients \\
\cline { 2 - 3 } & Urticaria vasculitis & $7(15 \%)$ \\
\cline { 2 - 3 } & Periungual telengiectasis & $6(13 \%)$ \\
\cline { 2 - 3 } & Raynaud's phenomenon & $4(8 \%)$ \\
\cline { 2 - 3 } & Livedo reticularis & $1(2 \%)$ \\
\cline { 2 - 3 } & Palpable purpura & $3(6 \%)$ \\
\hline
\end{tabular}




\section{Original Research Article}

As shown in Table 2, Malar rash was the most common skin finding in SLE patients (93\%) followed by oral ulcers.

Disease activity among SLE Patients was calculated as per the SLEDAI score at presentation and after 3 months of treatment. As shown in Figure 2, at presentation, majority of the SLE patients $(60 \%)$ had SLEDAI score $>20$ while at 3 months, majority of the patients (65\%) had SLEDAI score between 1-5 indicating improved outcome with treatment. The value of $t$ is -15.462 . The value of $p$ is $<0.00001$. The result is significant at $\mathrm{p} \leq 0.05$.

The mean value of SLEDAI score in the group with LE non -specific lesions was significantly higher compared to the group with LE-specific lesions $(\mathrm{P}<0.0001)$ thus indicating more active disease.

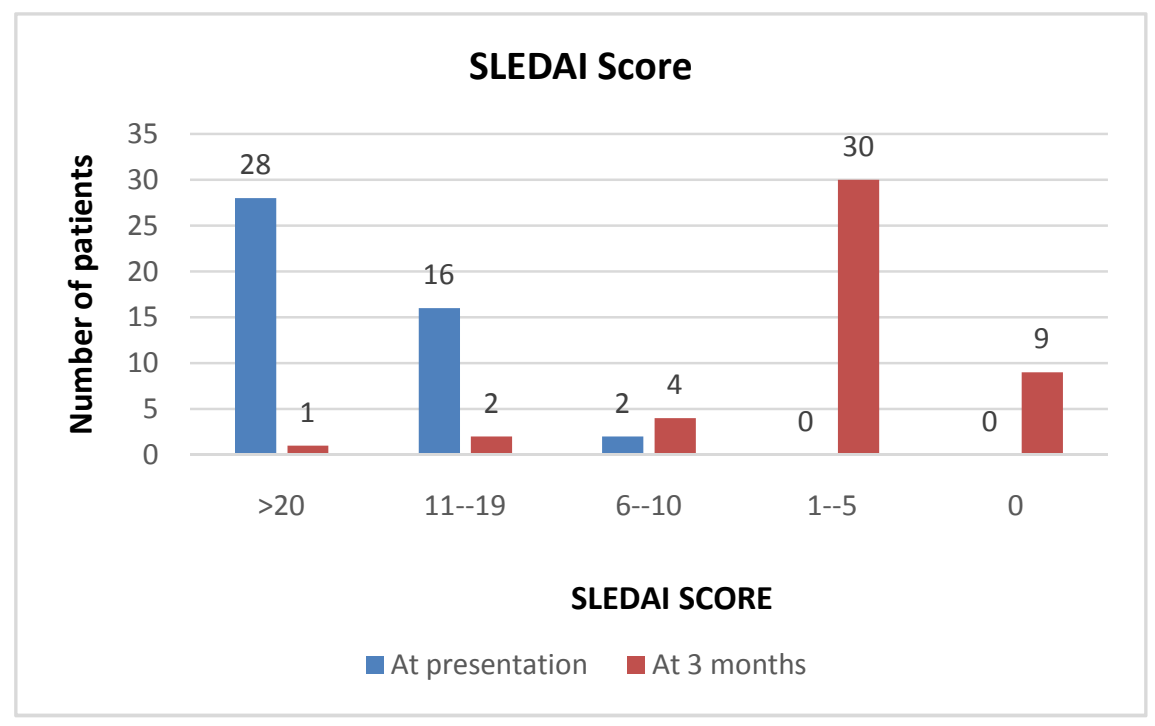

Figure-2: SLEDAI scores at presentation and at 3 months

SLICC ACR damage index was calculated at presentation for accumulated damage done by the disease over the years .Majority of patients $(60 \%, \mathrm{n}=28)$ had a score of 0 , indicating no evidence of a chronic damage due to SLE. $15 \%$ patients had damage indices values of 1 and 2 each indicating early damage. $6 \%$ patients $(n=3)$ had a damage index of $>5$ at presentation.

Rheumatoid Arthritis: The skin lesions in RA were grouped as RA specific and RA non-specific as shown in table 3. Rheumatoid nodules were the most common skin lesion, found in $56 \%$ of the RA patients included in the study while among the RA non-specific skin lesion, Palmar erythema was the most common skin lesion found in $30 \%$ of the patients.

At presentation as well at three months follow up, majority of the patients were in a state of low disease activity. But the number of patients in the state of remission (DAS 28 ESR Score <2.7) increased from 3 at presentation to 8 after three months of treatment as shown in Figure 3. In our study, 17 patients out of 23 RA patients (74\%) showed improvement in DAS 28 ESR score while 6 patients (26\%) did not show improvement with treatment.

Table-3: Skin lesions in RA

\begin{tabular}{|l|l|c|c|}
\hline \multirow{4}{*}{ RA specific lesion } & Skin lesion & Number & Percentage \\
\cline { 2 - 4 } & Rheumatoid nodules & 13 & $56 \%$ \\
\cline { 2 - 4 } & Rheumatoid vasculitis & 4 & $17 \%$ \\
\cline { 2 - 4 } & Pyoderma gangrenosum & 1 & $4 \%$ \\
\hline \multirow{4}{*}{ RA non -specific } & Urticaria & 1 & $4 \%$ \\
\cline { 2 - 4 } & Purpura & 1 & $4 \%$ \\
\cline { 2 - 4 } & Raynaud's phenomenon & 3 & $13 \%$ \\
\cline { 2 - 4 } & Palmar erythema & 7 & $30 \%$ \\
\hline
\end{tabular}




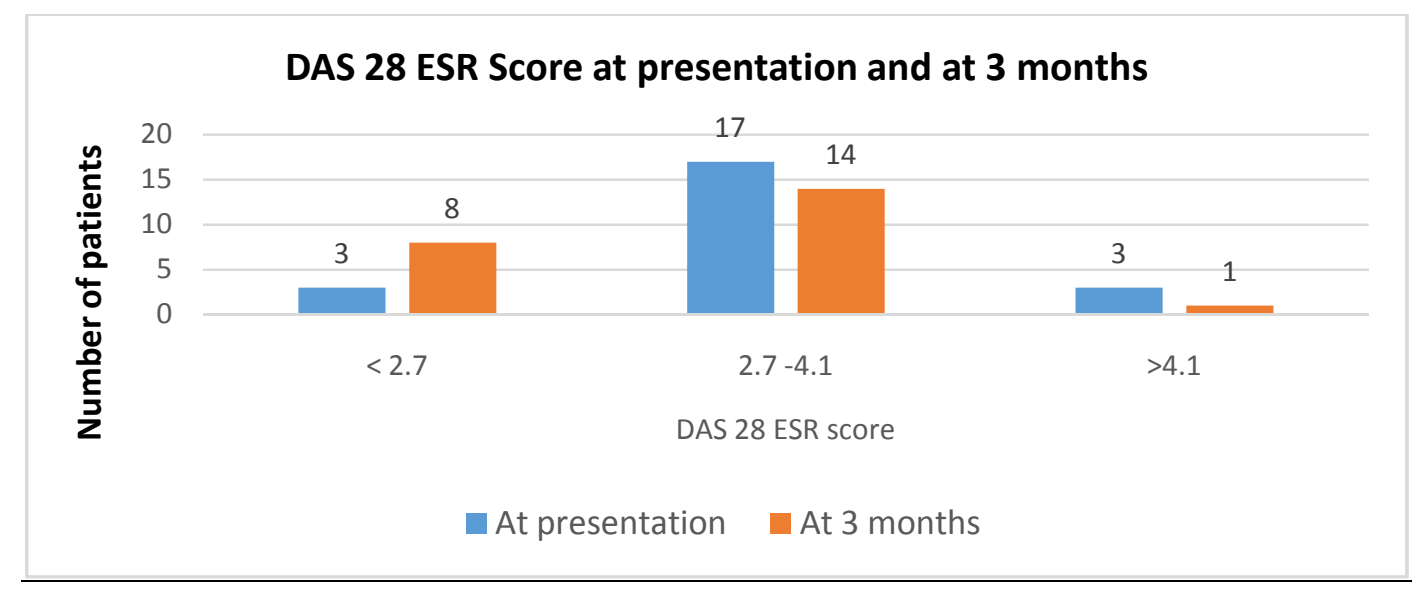

Figure-3: Comparison of DAS 28 ESR scores at presentation and at 3 months

Dermatomyositis: Among the group of Dermatomyositis patients, Heliotrope rash was the most common skin lesion, found in $75 \%$ patients $(n=3)$, followed by Gottron's papules seen in $50 \%$ patients $(n=2)$. Shawl sign and V sign were seen in $25 \%$ patients each.

Systemic Sclerosis: Of the 5 patients of scleroderma included in our study, 2 were of limited form, 2 were of diffuse form and 1 was of overlapping form. Raynaud's Phenomenon and Hidebound skin were the most common skin lesions seen in $100 \%$ patients, followed by Sclerodactyly which was seen in 4 patients. Calcinosis cutis, Microstomia, Salt and pepper appearance of skin were seen in 3 patients each (60\%). Digital ulcers and Telengiectasias were lesser common skin findings (seen in $20 \%$ patients, $n=1$ ).

The Modified Rodnan's Skin Score (MRSS) evaluation was performed in all patients with Scleroderma at presentation and at 3 months of treatment. 2 patients with evidence of ILD were given cyclophosphamide while 3 patients without ILD were given low dose steroid. The MRSS at presentation showed a mean value of $18 \pm 4.56$ points and after 3 months, mean value of MRSS was $16.4 \pm 5.2$ points (Table 4). The value of $t$ is -2.138 . The value of $p$ is 0.099 . Thus the difference in mean Modified Rodnan's score was not found to be statistically significant ( $p$ value $>0.05$ ), thus indicating not significant improvement in MRSS after 3 months of treatment.

Table-4: Modified Rodnan's skin score in 5 SSc patients

\begin{tabular}{|l|c|c|c|}
\hline & Type & At presentation & At 3 months \\
\hline Patient 1 & dSSc & 25 & 25 \\
\hline Patient 2 & dSSc & 21 & 19 \\
\hline Patient 3 & 1SSc & 15 & 15 \\
\hline Patient 4 & 1SSc & 12 & 10 \\
\hline Patient 5 & Overlapping & 17 & 13 \\
\hline Mean & & $\mathbf{1 8} \pm \mathbf{4 . 5 6}$ & $\mathbf{1 6 . 4 \pm 5 . 2}$ \\
\hline
\end{tabular}

\section{Discussion}

The mean age of presentation in our study was $38 \pm 10.4$ years with majority of patients being in the middle age group. This is consistent with other studies as most rheumatic diseases especially SLE which was the most frequent diagnosis in our patients, commonly present in the $4^{\mathrm{TH}}$ decade of life $[16,17]$. Most patients in our study were females $(93 \%)$ which is not surprising as most rheumatological conditions show a clear female preponderance as reported by previous studies $[17,18,19]$. The prerequisite for inclusion in the study was presentation of a new onset cutaneous lesion in rheumatological condition; hence all our patients had skin manifestation at presentation, followed by musculoskeletal complaints and constitutional symptoms. Previous studies have reported arthritis as more common initial manifestation seen in in $57 \%$ and $44 \%$ of the patients as reported by Malviya et al and Feng et al respectively [19,20], while a similar study from India by Kole et al reported higher prevalence of skin manifestations followed by arthritis consistent with our findings [5]. 


\section{Original Research Article}

Among the group of SLE patients, the skin lesions were further divided as LE specific and LE non specific [14]. Malar rash was the most common skin lesion seen in $93 \%$ patients similar to other previous studies. Vaidya et al reported an incidence of $53.18 \%$ while Aflak Rashid et reported malar rash in $66.64 \%$ patients in their study [7, 21]. Table 5 compares the skin manifestations in our study with 2 previous studies. We found a high incidence of Malar rash consistent with other studies by Kole et al and Aflak Rasheed et al [5,7].

Discoid type rash was much less frequently seen .Ina study done by Kapadia et al also the incidence of Discoid rash was much lower [22]. We did not find any cases of Generalized maculopapular eruptions or Subacute Cutaneous Lupus Erythematosis (SCLE) in our study. Among the group with LE nonspecific lesions Alopecia and oral ulcers were most frequently seen.

The correlation between the type of skin manifestation and disease activity and the correlation between the number of skin lesion types and disease activity using SLEDAI score was studied by RD Zecević et al in 66 SLE patients [23]. It was found that LE non-specific lesions were associated higher disease activity as compared to LE- specific skin lesions as measured by SLEDAI score. Also, the number of different types of skin lesion proved to co-relate with disease activity so that the severity of the disease increases with the number of lesions. Similar results were seen in our study with the group with LE non specific lesions showing more active disease as measured by SLEDAI score. The study done by Kole et al from eastern India also describes similar results with LE non specific lesions indicating more active disease [5].

Table-5: Comparison of Skin lesions in SLE Patients in our study vs previous studies

\begin{tabular}{|l|l|c|c|c|}
\hline \multirow{5}{*}{ LE specific } & Malar rash & $\begin{array}{c}\text { Kole et al [5] } \\
\mathbf{N = 1 5 0}\end{array}$ & $\begin{array}{c}\text { Aflak Rasheed } \\
\text { et al [7] N=125 }\end{array}$ & Present study \\
\cline { 2 - 5 } & Photosensitive dermatitis & $120(80 \%)$ & $83(66.64 \%)$ & $43(93.47 \%)$ \\
\cline { 2 - 5 } & Generalised maculopapular rash & $75(50 \%)$ & $61(48.8 \%)$ & $21(46.65 \%)$ \\
\cline { 2 - 5 } & Discoid rash & $30(20 \%)$ & $23(18.4 \%)$ & - \\
\cline { 2 - 5 } specific & Subacute cutaneous lesions & $5(3.34 \%)$ & $6(4.8 \%)$ & NA \\
\cline { 2 - 5 } & Lupus profundus & $5(3.34 \%)$ & NA & $3(6.52 \%)$ \\
\cline { 2 - 5 } & Non-scarring alopecia & $130(86.67 \%)$ & NA & $39(84.78 \%)$ \\
\cline { 2 - 5 } & Scarring alopecia & $10(6.67 \%)$ & $39(31.2 \%)$ & NA \\
\cline { 2 - 5 } & Oral ulcer & $85(56.67 \%)$ & $39(31.2 \%)$ & $39(84.78 \%)$ \\
\cline { 2 - 5 } & Vasculitic lesions & $50(33.34 \%)$ & $50(40 \%)$ & NA \\
\cline { 2 - 5 } & Raynaud's Phenomenon & $10(6.67 \%)$ & $3(2.4 \%)$ & $4(8.6 \%)$ \\
\cline { 2 - 5 } & Periungual telengiectasis & $2(1.34 \%)$ & NA & $6(13.64 \%)$ \\
\cline { 2 - 5 } & Pyoderma gangrenosum & $2(1.34 \%)$ & NA & NA \\
\cline { 2 - 5 } & Nail fold infarcts & $2(1.34 \%)$ & NA & NA \\
\cline { 2 - 5 } & Livedo reticularis & NA & $5(4 \%)$ & NA \\
\cline { 2 - 5 } & Digital gangrene & NA & $3(2.4 \%)$ & No.17\%) \\
\hline
\end{tabular}

Among the group of 23 rheumatoid arthritis patients, 13 patients with prolonged disease duration of 8-10 years were observed to have cutaneous changes. The skin lesions were divided as RA specific and RA nonspecific [15]. Rheumatoid nodule was the most common skin lesion found in $56.52 \%$ patients followed by rheumatoid vasculitis (17\%). Even in previous studies, Rheumatoid nodule was seen as most frequent skin involvement in RA patients [24] which has been reported especially in long standing and more severe RA cases. The incidence of RA vasculitis was higher as compared to the study by Bhanuprakash et al [17], who reported an incidence of 5.8\% compared to $17.4 \%$ in our study. In a autopsy study by Suzuki et al in 1994 the incidence of Vasculitis was found to be 30\% among 81 autopsied RA patients [25] while a more recent study in 2015 by Cojocaru $M$ et al describes a much lower incidence of 2-5\% [26]. The decrease in incidence may be attributable to the treatment advances made with the advent of targeted Biologics in treatment of RA. Raynauds phenomenon was seen in $13 \%$ patients. This finding is consistent with previous studies reporting an incidence of about $12-13 \%$ [27]. 
The most commonly detectable cutaneous features in dermatomyositis were the violaceous macular erythema- the heliotrope rash and Gottron's papules found in $75 \%$ and $50 \%$ patients in the study population. The age group in the study population was 45-55 years and there was a clear female preponderance with a 3:1 female to male ratio as reported in earlier studies [28]. We could not find any juvenile DM in our study. Mean age of study population was 51.4 year which was nearly comparable to study done by Parodi et al [28]. Cutaneous features, such as the mechanic's hands, vasculitis, lichen planus-like papules and livedo reticularis were not seen in the study population. Raynaud's phenomenon is reported to occur in $0-20 \%$ of individuals with DM [28], but was not found in our study. In patients with systemic sclerosis, Raynaud's phenomenon was present in all patients in our study, similar to previous study by Fernanda Guidolin et al [29], while the percentage of microstomia and calcinosis was found to be higher in the present study (60\% each) compared to $31 \%$ and $12 \%$ respectively. Previous other studies have also documented a high prevalence of Raynauds Phenomenon in patients with Systemic sclerosis and it is more commonly seen in males and may be the presenting complaint in as many as $70 \%$ of patients [30].

Modified Rodnan's Skin Score (MRSS) was calculated for all SSc patients at baseline and again at 3 months follow up. There was no significant improvement in MRSS after 3 months of treatment. A previous study done by Patrícia Andrade de Macedo et al [31] in Brazilevaluated effectiveness of Cyclophosphamide in the treatment of severe cutaneous involvement in systemic sclerosis showed that there was significant reduction in MRSS after 18 months of treatment. However, since the follow up period in our study was only 3 months, it may be inadequate to comment on the potential improvement in the long run in these patients on Cyclophosphamide therapy.

\section{Conclusions}

Skin lesions are an important diagnostic clue in Rheumatological diseases and help in classifying the diseases. Malar rash in SLE, Rheumatoid Nodules in RA, Sclerodactyly in systemic sclerosis and Heliotrope rash in Dermatomyositis are the most common skin manifestations seen. SLE is the most common Rheumatological condition presenting with cutaneous manifestations. Patients with LE specific lesions have lower disease activity as compared to LE nonspecific lesions.

At 3 months follow up the response to treatment is good in SLE patients with reduction in SLEDAI scores and also in RA patients with reduction in DAS 28 ESR scores. Systemic sclerosis patients failed to show significant improvement in skin scores (MRSS) after 3 months of treatment.

Contributions by Authors: All authors have contributed to the intellectual content of this paper and have met the following 3 requirements: (a) significant contributions to the conception and design of the study, data collection, analysis and interpretation; (b) drafting and critically revising the article and (c) final approval of the published article.

What does this study add to existing knowledge (Learning Points): A new onset skin manifestation often helps in diagnosing and classifying various rheumatic diseases. In the group of SLE patients LE specific lesions are usually highlighted where as our study found higher disease activity Index (SLEDAI score) amongst patients with LE nonspecific lesions.
Thusemphasizing the point that any such lesions appearing, should alert the physician towards worsening of the disease activity while on treatment. Among the group of systemic sclerosis patients long term studies are needed to evaluate outcome of the cutaneous features after treatment.

\section{Funding: Nil, Conflict of interest: None Permission of IRB: Yes}

\section{References}

1. Hochberg MC. Updating the American College of Rheumatology revised criteria for the classification of systemic lupus erythematosus. Arthritis Rheum. 1997 Sep; 40(9):1725. DOI:10.1002/1529-0131(199709) 40:9 \& 1t;1725::AID-ART29\&gt;3.0.CO;2-Y

2. Tan EM, Cohen AS, Fries JF, et al. The 1982 revised criteria for the classification of systemic lupus erythematosus. Arthritis Rheum. 1982 Nov; 25 (11): 1271-7.

3. Wananukul S, Watana D, Pongprast P. Cutaneous manifestations of childhood systemic lupus erythematosus. Pediatr Dermatol. 1998;15:342-6.

4. O'Quinn SE, Cole J, Many H. Problems of disability and rehabilitation in patients with chronic skin diseases. Arch Dermatol. 1972 Jan;105(1):35-41.

5. Kole AK, Ghosh A. Cutaneous manifestations of systemic lupus erythematosus in a tertiary referral center. Indian J Dermatol. 2009; 54 (2) :132-6. doi: 10.4103 / 0019-5154.53189. 


\section{Original Research Article}

6. Lee $\mathrm{HH}^{1}$, Song IH, Friedrich $\mathrm{M}$, et al. Cutaneous side-effects in patients with rheumatic diseases during application of tumour necrosis factor-alpha antagonists. Br J Dermatol. 2007 Mar;156(3):486-91. DOI:10. 1111/ j.1365-2133.2007.07682.x

7. Aflak Rasheed, Shazia Rasul, Abdul Hameed. Prevalence of usual and unusual skin manifestations of systemic lupus erythematosus in a tertiary care hospital. Journal of Pakistan Association of Dermatologists. 2016;26 (2):118-122

8. Laman SD, Provost TT. Cutaneous manifestations of lupus erythematosus. Rheum Dis Clin North Am. 1994 Feb;20(1):195-212.

9. Aletaha D, Neogi T, Silman AJ, et al. 2010 rheumatoid arthritis classification criteria: an American College of Rheumatology/European League Against Rheumatism collaborative initiative. Ann Rheum Dis. 2010 Sep ;69 (9): 1580-8. doi: 10.1136/ ard . 2010. 138461 .

10. Gladman DD, Ibañez D, Urowitz MB. Systemic lupus erythematosus disease activity index 2000. J Rheumatol. 2002 Feb;29(2):288-91.

11. Gladman DD, Urowitz MB, Goldsmith $\mathrm{CH}$, et al. The reliability of the Systemic Lupus International Collaborating Clinics/American College of Rheumatology Damage Index in patients with systemic lupus erythematosus. Arthritis Rheum. 1997 May;40(5): 80913. DOI : 10.1002 / 1529-0131 (199705) 40:5 \& lt;809:: AID-ART5\&gt;3.0.CO;2-X

12. Prevoo ML, van 't Hof MA, Kuper HH, et al. Modified disease activity scores that include twentyeight-joint counts. Development and validation in a prospective longitudinal study of patients with rheumatoid arthritis. Arthritis Rheum. 1995 Jan;38 (1): 44-8.

13. Clements PJ, Lachenbruch PA, Seibold JR, et al. Skin thickness score in systemic sclerosis: an assessment of interobserver variability in 3 independent studies. J Rheumatol. 1993 Nov;20 (11):1892-6.

14. Gilliam JN, Sontheimer RD. Skin manifestations of SLE. Clin Rheum Dis. 1982 Apr;8(1):207-18.

15. Yamamoto T. Cutaneous manifestations associated with rheumatoid arthritis. Rheumatol Int. 2009 Jul;29 (9): 979-88. doi: 10.1007/s00296-009-0881-z. Epub 2009 Feb 26.
16. Masi AT, Kaslow RA. Sex effects in systemic lupus erythematosus: a clue to pathogenesis. Arthritis Rheum. 1978 May; 21(4):480-4.

17. Prakash B, CA Jayashankar, Shivalingappa VM, Bhakthavatsalam SA, Chandrashekar K. Cutaneous Manifestations of Rheumatoid Arthritis. IJRCI. 2015;3 (1): OA2. DOI: 10.15305/ijrci/v3i1/107

18. Das NK, Dutta RN, Sengupta SR. Skin lesions in lupus erythematosus: a marker of systemic involvement. Indian J Dermatol. 2011 Sep-Oct;56(5):537-40. doi: 10.4103/0019-5154.87150.

19. Malaviya AN, Singh RR, Kumar A, et al. Systemic lupus erythematosus in northern India: a review of 329 cases. J Assoc Physicians India. 1988 Aug;36(8):476$80,484$.

20. Feng PH, Boey ML. Systemic lupus erythematosus in Chinese: the Singapore experience. Rheumatol Int. 1982;2(4):151-4.

21. Vaidya S, Samant RS. SLE-review of two hundred and twenty patients. J India Rheumatol Assoc 1997;5:14-8.

22. Kapadia N, Haroon TS. Cutaneous manifestations of systemic lupus erythematosus: study from Lahore, Pakistan. Int J Dermatol. 1996 Jun;35(6):408-9.

23. Zecević RD, Vojvodić D, Ristić B, et al. Skin lesions--an indicator of disease activity in systemic lupus erythematosus? Lupus. 2001;10(5):364-7. DOI: 10. $1191 / 096120301675962535$

24. Sayah A, English JC 3rd. Rheumatoid arthritis: a review of the cutaneous manifestations. DOI:10.1016/ j.jaad.2004.07.023

25. Suzuki A, Ohosone Y, Obana M, et al. Cause of death in 81 autopsied patients with rheumatoid arthritis. J Rheumatol. 1994 Jan;21(1):33-6.

26. Cojocaru M, Cohocaru IM, Chico B. New insight into the rheumatoid vasculitis. Rom J Intern Med. 2015 Apr-Jun;53(2):128-32. DOI:10.1515/rjim-2015-0017

27. Sharma A, Albert D (2015) Dermatologic Manifestations of Rheumatoid Arthritis. Rheumatology (Sunnyvale) 5: 168. doi: 10.4172/2161-1149.1000168. 


\section{Original Research Article}

28. Parodi A, Caproni M, Marzano AV, et al. Dermatomyositis in 132 patients with different clinical subtypes: cutaneous signs, constitutional symptoms and circulating antibodies. Acta Derm Venereol. 2002;82 (1):48-51.

29. Guidolin, Fernanda, Esmanhotto, Letícia, Magro, Carlos Eduardo, Silva, Marilia Barreto, \&Skare, Thelma L.. (2005). Prevalence of cutaneous findings in systemic sclerosis patients: experience of a teaching hospital. Anais Brasileiros de Dermatologia, 80(5), 481-486.
30. Tager RE, Rikly M. Clinical and laboratory manifestations of systemic sclerosis (scleroderma) in black South Africans. Rheumatology. 1999; 38:397400. PMID: 10371275 doi: 10.1093 /rheumatology/38.5.397.

31. Patrícia Andrade de Macedo, Cláudia Teresa Lobato Borges, Romy Beatriz Christmann de Souza: Cyclophosphamide: effective in the treatment of severe cutaneous involvement in systemic sclerosis, Bras J Rheumatol 2009;49 (3):265-75 DOI: 10.1590/S048250042009000300008

\section{How to cite this article?}

Sonawale A., Sabnis N., Bankar N. Study of new onset cutaneous manifestations in Rheumatic Diseases. Int J Med Res Rev 2018; 6(08): 510-518. doi:10.17511/ijmrr.2018.i08.17. 